\title{
Thyroid dysfunction and insulin resistance in patients with nonalcoholic fatty liver disease

\author{
Maha Assem ${ }^{\mathrm{a}}$, May Fawzi ${ }^{\mathrm{a}}$, Alhosaeyn Ibrahim $^{\mathrm{b}}$, Aasem Saif ${ }^{\mathrm{a}}$
}

aDepartment of Internal Medicine, Faculty of Medicine, Cairo University, 'Department of Gastroenterology, Ahmed Maher Hospital, Cairo, Egypt

Correspondence to Maha Assem Hussein, Department of Internal Medicine, Faculty of Medicine, Cairo University, Egypt. e-mail: maha.assem@hotmail.com

Received 10 January 2018

Accepted 5 May 2018

The Egyptian Journal of Internal Medicine 2018, 30:97-102

\begin{abstract}
Introduction
Nonalcoholic fatty liver disease (NAFLD) is a common cause of impaired liver functions. It is associated with hepatic and adipose tissue insulin resistance (IR) as well as decreased whole-body insulin sensitivity. Thyroid hormones are important for the intrahepatic metabolism of lipids. Thyroid disorders have been associated with IR owing to various mechanisms such as altered insulin secretion and lipid levels.
\end{abstract}

Aim

The aim of our study was to assess thyroid functions, thyroid autoimmunity, and IR in nondiabetic patients with NAFLD.

\section{Materials and methods}

The study was conducted on 90 nondiabetic participants (60 patients with NAFLD and 30 participants with normal liver). Both groups were sex matched. Ultrasonography was used to categorize the study participants into NAFLD and normal liver groups. Thyroid functions and thyroid peroxidase antibody were assessed in all participants. Homeostatic model assessment (HOMA IR) was used to assess IR in the study population.

Results

Our results showed a significantly higher HOMA IR in the NAFLD compared with the normal liver group $(P<0.001)$. Moreover, significantly higher percentage of patients with NAFLD have IR (HOMA IR) compared with the normal liver group (56.7 vs. $6.7 \%$, respectively; $P<0.001)$. Our results also showed higher percentage of thyroid dysfunction in patients with NAFLD compared with individuals without NAFLD $(P<0.02)$. Overall, $22(36.7 \%) 60$ patients in the NAFLD group versus three $(10 \%)$ of 30 patients in the non-NAFLD group had subclinical hypothyroidism. Thyroid functions did not show any statistically significant correlations with IR, but thyroid peroxidase antibody showed significant positive correlation with $\operatorname{IR}(P<0.02)$ within the total study population.

\section{Conclusion}

There is an increased prevalence of subclinical hypothyroidism in nondiabetic patients with NAFLD who also have higher IR. The role of thyroid autoimmunity in this relationship needs further assessment.

\section{Keywords:}

autoimmunity, insulin resistance, nonalcoholic fatty liver, thyroid

Egypt J Intern Med 30:97-102

(C) 2018 The Egyptian Journal of Internal Medicine

$1110-7782$

\section{Introduction}

Nonalcoholic fatty liver disease (NAFLD) is the most common cause of liver disease worldwide, increasing in parallel with that of obesity and diabetes [1]. It is a complex disease that is modulated by numerous mechanisms including metabolic, genetic, environmental, and gut microbial factors [1]. NAFLD is strongly associated with both hepatic and adipose tissue insulin resistance (IR) as well as reduced whole-body insulin sensitivity [2]. Thyroid hormones are important for the intrahepatic metabolism of lipids including $\beta$-oxidation of fatty acid and delivery of fatty acids to mitochondria [3]. Many data suggest that hypothyroidism is one of the risk factors for NAFLD [4]. Thyroid disorders, including both hypothyroidism and hyperthyroidism, have been associated with IR owing to various mechanisms such as altered insulin secretion and lipid levels. Although hypothyroidism and hyperthyroidism constitute an IR state, more studies need to be done to clarify the underlying pathogenic mechanism [5].

We studied the relationship between thyroid functions and IR in nondiabetic patients with NAFLD. This relationship may provide valuable data for better understanding the effect of each of those three distinct disorders (thyroid dysfunctions, IR, and NAFLD) on the

\footnotetext{
This is an open access journal, and articles are distributed under the terms of the Creative Commons Attribution-NonCommercial-ShareAlike 4.0 License, which allows others to remix, tweak, and build upon the work non-commercially, as long as appropriate credit is given and the new creations are licensed under the identical terms.
} 
other two entities, which could be of value in establishing a hypothesis for a mutual prophylactic or curative plan for them.

\section{Materials and methods}

A cross-sectional analytical study was conducted on a total of 98 participants who visited the Ultrasound Unit of Internal Medicine Department at Kasr Al Aini Hospital, Cairo University, for a routine health check-up between January 2016 and March 2016. Overall, 90 participants were involved in the study. Each was chosen based on the following criteria.

\section{Inclusion criteria}

Patients presented for routine abdominal ultrasound and found to have fatty liver, with history free of alcohol consumption in the age group 18-60 years old and having BMI greater than or equal to $18.5 \mathrm{~kg} / \mathrm{m}^{2}$ and less than or equal to $40 \mathrm{~kg} / \mathrm{m}^{2}$ were included.

\section{Exclusion criteria}

Patients with any liver diseases such as liver cirrhosis, autoimmune or viral-induced hepatitis, and hepatobiliary malignancies, patients with history of thyroid disease, diabetic patients, and patients with BMI greater than $40 \mathrm{~kg} / \mathrm{m}^{2}$ were excluded.

The study was approved by Cairo University ethical committee and review board. All the patients and controls who participated in the study provided written informed consents.

These 90 participants were classified into two groups and were sex matched. Group 1 included 60 participants with ultrasound evidence of NAFLD (30 males and 30 females), and group 2 included 30 participants with normal liver ultrasound finding (15 males and 15 females). All participants were subjected to the following:

(1) Clinical examination: full history, general and systemic examination. BMI was calculated as body weight in $\mathrm{kg}$ divided height square in meters.

(2) Ultrasonographic evaluation of the liver: the ultrasound examinations were performed by an experienced consultant who was unaware of the clinical and laboratory data. Liver ultrasound was done by conventional B-mode with a convex $3.5 \mathrm{MHz}$ probe [alanine aminotransferase (ALT) HDI ultramark machine, Philips]. All participants were examined by the same operator and the same ultrasound device.
NAFLD was defined as the presence of the following four ultrasonographic criteria: hepatorenal echo contrast, liver brightness, deep attenuation, and vascular blurring in the absence of (a) seropositivity for hepatitis B surface antigen or antibody to hepatitis $\mathrm{C}$ virus; (b) alcohol consumption; (c) history of other causes of liver disease; and (d) medications known to produce fatty liver disease during the last 6 months before the study [6].

(1) Laboratory investigations: blood samples were obtained from each participant after overnight fasting of greater than $8 \mathrm{~h}$. Laboratory studies included fasting blood sugar in $\mathrm{mg} / \mathrm{dl}$, complete blood count, urea, and creatinine; serum total cholesterol (TC), high-density lipoproteincholesterol, low-density lipoprotein (LDL)cholesterol, and triglycerides (TGs); serum levels of ALT, aspartate aminotransferase (AST), total bilirubin, total proteins and albumin; coagulation profile [Prothrombin concentration (PC) and international normalized ratio]; and antihepatitis $\mathrm{C}$ antibody, hepatitis B virus surface antigen, and hepatitis B surface antibody. Fasting insulin level, thyroid function tests [free $\mathrm{T}_{3}$, free $\mathrm{T}_{4}$, and thyroidstimulating hormone (TSH)], and thyroid peroxidase antibody (TPO-Ab) were also assessed. Homeostatic model assessment (HOMA)-IR was used to evaluate IR. It was calculated using the following formula: fasting serum insulin $(\mu \mathrm{U} /$ $\mathrm{ml}) \times$ fasting plasma glucose $(\mathrm{mmol} / \mathrm{l}) / 22.5$.The physiological value of the index is 1.0. Values above 1.9 indicate IR [7]. The thyroid profile (free $\mathrm{T}_{3}$, free $\mathrm{T}_{4}$, and $\mathrm{TSH}$ ) was assessed by enzyme-linked immunosorbent assay (ELISA) technique. The cutoff values for each of them used to assess the thyroid status were as follows:

(a) Free $\mathrm{T}_{3}(1.4-4.2 \mathrm{pg} / \mathrm{dl})$.

(b) Free $\mathrm{T}_{4}(0.65-1.97 \mathrm{ng} / \mathrm{dl})$.

(c) $\mathrm{TSH}(0.4-6 \mu \mathrm{IU} / \mathrm{ml})$.

Fasting insulin level and TPO-Ab were was assessed by ELISA technique.

$\mathrm{TPO}-\mathrm{Ab}$ index interpretation was as follows:

(1) $0.9-1.1$ Borderline positive.

(2) Greater than 1.1 detectable TPO antibody by ELISA.

\section{Converting of $A b$ index to $\mathrm{IU} / \mathrm{ml}$}

As an option, TPO-Ab index may be converted to IU/ $\mathrm{ml}$ by multiplying $\mathrm{Ab}$ index value by 50 .

International units may then be interpreted as follows: 
(1) Less than $45 \mathrm{lU} / \mathrm{ml}$ : negative.

(2) 45-55 IU/ml: borderline positive.

(3) Greater than 55: positive.

\section{Statistical method}

Data were statistically analyzed using the statistical package for the social sciences software program, version 23 (IBM SPSS Statistics for Windows, Version 23.0., IBM Corp. Armonk, New York, USA). Comparison between groups was performed using independent sample $t$-test for quantitative variables and $\chi^{2}$-test for qualitative ones. Spearman's correlation coefficients ( $r$ values) were calculated to signify the association between different quantitative variables. $P$ values less than 0.05 were considered statistically significant, and less than 0.01 were considered highly significant.

\section{Results}

A cross-sectional study with a total of 45 pairs of sexmatched health checkup participants (60 cases with NAFLD vs. 30 controls with normal liver ultrasound) was conducted. The mean levels of each of age, BMI, ALT, AST, TC, TGs, LDL, fasting blood glucose, fasting insulin, and HOMA IR were significantly higher in patients with NAFLD than in those with normal liver ultrasound findings, with $P$ less than 0.001 (Table 1). There was a statistically significant higher prevalence of thyroid dysfunction (whether subclinical hyperthyroidism or subclinical hypothyroidism) among patients with fatty liver than those with normal liver ultrasound (5 and $3.3 \%$ of the participants, respectively, showed subclinical hyperthyroidism and 36.7 and $10 \%$ of the participants, respectively, showed subclinical hypothyroidism, with $P<0.02)$. Only four $(6.7 \%)$ patients in the NAFLD group were positive for anti-TPO antibodies versus none in the normal liver group $(P=0.297)$. Moreover, significantly higher percentages of patients with NAFLD have insulin resistance (HOMA IR) compared with the normal liver group (56.7 and 6.7\%, respectively; $P<0.001$ ). A significantly higher percentage of patients with NAFLD had hepatomegaly compared with the normal liver group $(P<0.001$; Table 2$)$.

\section{The relationship between homeostatic model} assessment insulin resistance and other variables

The Spearman correlation coefficient for the relationship between the HOMA IR as a dependent variable and the other independent variables in the study showed that there was a significant positive correlation between HOMA IR and liver enzymes (ALT and AST), TC, and TGs in both groups, but it was significantly positively correlated with LDL in
Table 1 Clinical and laboratory data of the study groups

\begin{tabular}{|c|c|c|c|}
\hline & $\begin{array}{c}\text { NAFLD } \\
(n=60) \\
\text { (group I) } \\
\text { (mean } \pm S D)\end{array}$ & $\begin{array}{c}\text { Normal } \\
(n=30) \\
\text { (group II) } \\
(\text { mean } \pm S D)\end{array}$ & $P$ value \\
\hline Age (years) & $37.6 \pm 8.8$ & $29.1 \pm 9.1$ & $<0.001$ \\
\hline BMI & $29.6 \pm 4.1$ & $26.5 \pm 4.1$ & 0.001 \\
\hline ALT (IU/I) & $69.9 \pm 21.2$ & $30.5 \pm 16.0$ & $<0.001$ \\
\hline AST (IU/I) & $64.9 \pm 22.4$ & $30.0 \pm 14.8$ & $<0.001$ \\
\hline $\begin{array}{l}\text { Total cholesterol } \\
(\mathrm{mg} / \mathrm{dl})\end{array}$ & $225.1 \pm 29.0$ & $136.6 \pm 42.5$ & $<0.001$ \\
\hline TGs (mg/dl) & $161.5 \pm 18.9$ & $81.2 \pm 39.4$ & $<0.001$ \\
\hline LDL (mg/dl) & $166.2 \pm 27.2$ & $84.1 \pm 42.2$ & $<0.001$ \\
\hline $\mathrm{HDL}(\mathrm{mg} / \mathrm{dl})$ & $42.9 \pm 4.6$ & $43.1 \pm 2.8$ & 0.609 \\
\hline FBG (mg/dl) & $5.3 \pm 0.4$ & $4.7 \pm 0.5$ & $<0.001$ \\
\hline $\begin{array}{l}\text { Fasting insulin }(\mu \mathrm{U} / \\
\mathrm{ml})\end{array}$ & $8.5 \pm 2.0$ & $6.2 \pm 1.5$ & $<0.001$ \\
\hline HOMA IR & $2.00 \pm 0.57$ & $1.29 \pm 0.34$ & $<0.001$ \\
\hline FT3 (pg/dl) & $1.82 \pm 0.52$ & $1.72 \pm 0.36$ & 0.473 \\
\hline FT4 (ng/dl) & $1.04 \pm 0.19$ & $1.09 \pm 0.26$ & 0.914 \\
\hline TSH $(\mu \mathrm{IU} / \mathrm{ml})$ & $3.38 \pm 2.95$ & $1.96 \pm 1.92$ & 0.054 \\
\hline $\begin{array}{l}\text { TPO-AB index (IU/ } \\
\text { ml) }\end{array}$ & $0.31 \pm 0.53$ & $0.18 \pm 0.08$ & 0.637 \\
\hline
\end{tabular}

ALT, alanine aminotransferase; AST, aspartate aminotransferase, HDL, high-density lipoprotein; HOMA IR, homeostatic model assessment insulin resistance; LDL, low-density lipoprotein; NAFLD, nonalcoholic fatty liver disease; TG, triglyceride; TSH, thyroid-stimulating hormone; TPO-Ab, thyroid peroxidase antibody.

the group of normal liver only. HOMA IR was significantly positively correlated with fasting insulin level in both groups, but it was significantly positively correlated with fasting blood glucose levels in the group of fatty liver only.

TSH showed statistically significant positive correlation with age in the NAFLD group $(P<0.025)$. No other significant correlations were found for TSH with other variables in NAFLD group or all the variables in the normal liver group.

Positive TPO-Abs were found in only four participants (all of them had NAFLD and high IR). The four participants who had positive TPO-Ab also showed higher mean levels of the TC, lipoprotein, fasting blood glucose, and fasting insulin level than other participants. The prevalence of IR was higher in participants with positive TPO-Ab (100\%) than in those with negative TPO-Ab (37.2\%).

\section{Discussion}

Our study was conducted on 90 participants categorized by ultrasonography into two groups: 60 participants with NAFLD and 30 participants with normal liver. Both groups were sex matched. HOMA IR was used to assess IR in the study population. Thyroid functions and TPO$\mathrm{Ab}$ were used to assess the thyroid status. 
Table 2 Results of thyroid functions, antithyroid peroxidase antibody, homeostatic model assessment insulin resistance, and liver ultrasonography in the study groups

\begin{tabular}{|c|c|c|c|}
\hline & \multicolumn{2}{|c|}{ Liver texture $[n(\%)]$} & \multirow[t]{2}{*}{$P$ value } \\
\hline & NAFLD $(n=60)$ (group I) & Normal $(n=30)$ (group II) & \\
\hline \multicolumn{4}{|l|}{ Sex } \\
\hline Male & $30(50.0)$ & $15(50.0)$ & 1.0 \\
\hline Female & $30(50.0)$ & $15(50.0)$ & \\
\hline \multicolumn{4}{|l|}{ HOMA IR interpretation } \\
\hline IR & $34(56.7)$ & $2(6.7)$ & $<0.001$ \\
\hline Normal & $26(43.3)$ & $28(93.3)$ & \\
\hline \multicolumn{4}{|l|}{ Thyroid function interpretation } \\
\hline Subclinical hyperthyroid & $3(5.0)$ & $1(3.3)$ & 0.022 \\
\hline Subclinical hypothyroid & $22(36.7)$ & $3(10.0)$ & \\
\hline Normal thyroid & $35(58.3)$ & $26(86.7)$ & \\
\hline \multicolumn{4}{|l|}{ TPO-Ab interpretation } \\
\hline Positive & $4(6.7)$ & $0(0.0)$ & 0.297 \\
\hline Negative & 56 (93.3) & $30(100.0)$ & \\
\hline \multicolumn{4}{|c|}{ Hepatomegaly (by ultrasonography) } \\
\hline Yes & $47(78.3)$ & $6(20.0)$ & $<0.001$ \\
\hline No & $13(21.7)$ & $24(80.0)$ & \\
\hline
\end{tabular}

HOMA IR, homeostatic model assessment insulin resistance; NAFLD, nonalcoholic fatty liver disease; TPO-Ab, thyroid peroxidase antibody.

In our study, IR was detected in 36 participants ( $40 \%$ of the study population). They were distributed as follows: $56.6 \%$ of total participants with NAFLD and $6.7 \%$ of those with normal liver ultrasonography finding. Our results showed a significantly higher HOMA IR in the NAFLD compared with the normal liver group $(P<0.001)$. The mean values of both ALT and AST as well as the mean values of both TC and TGs were significantly higher in participants with IR than those with normal insulin sensitivity. HOMA IR showed a statistically significant correlation with liver enzymes as well as cholesterol and TG levels in both groups.

In patients with non alcoholic steatohepatitis (NASH), it is controversial whether the liver disease plays a primary role in the genesis of hyperinsulinemia and IR or is a consequence of IR as a part of the metabolic syndrome. Previous studies on the association between IR and NASH enrolled patients with variable but often severe degrees of steatohepatitis and fibrosis. Under these conditions, hepatocellular damage and portal systemic shunting can per-se impair hepatic degradation, leading to chronic hyperinsulinemia and subsequent tissue $\mathrm{IR}$, as is the case in cirrhosis and other chronic liver diseases [8]. A significant study of insulin insensitivity involved a group of patients with both NAFL and NASH who were chosen because they were neither diabetic nor obese and had normal 2-h oral glucose tolerance test results. The study included 30 lean patients with NAFL, 21 with NASH and nine with pure fatty liver. Insulin sensitivity testing revealed all of the patients had at least one clinical sign of metabolic syndrome. Their fasting insulin levels, lipid profiles, and waist-to-hip ratios were significantly different from a healthy control group and similar to a comparison group of type 2 diabetics. These studies indicate that IR, apparent in NASH, may exist even without, and possibly preceding, apparent glucose intolerance or obesity [9].

Our findings are in agreement with many population studies which showed that nearly all of the patients with NAFLD examined were IR according to the homeostasis model assessment (HOMA) [10]. Bugianesi et al. [11] reported that the presence of liver steatosis (assessed using computerized tomography criteria) in patients with type 2 diabetes is associated with more severe IR.

Over the past decade, the association between thyroid dysfunction and NAFLD has increasingly become a focus of research. After some controversial reports, numerous studies have confirmed an association between thyroid function and NAFLD [12].

Our study did not report any clinical thyroid disorder in both groups. There were no significant differences in FT3 and FT4 levels between the two groups. Subclinical hyperthyroidism was detected in 5\% of the NAFLD group versus $3.3 \%$ of the normal group. However, subclinical hypothyroidism represented $36.7 \%$ of the NAFLD group versus $10 \%$ of the normal group. It has been reported that hypothyroidism was more common in patients with NASH compared with patients with NAFLD. This finding remained statistically significant 
after adjusting for other variables including age, diabetes, dyslipidemia, and hypertension but not sex $[12,13]$. A study, which used liver biopsy and the NAFLD activity score to distinguish NASH from NAFLD provided additional evidence for the association between the severity of liver fatty infiltration and hypothyroidism. Pagadala et al. [14] suggested, in addition, that the TSH concentration is associated with the severity of the hepatic steatosis. Therefore, there might be a possible association between thyroid dysfunction and NAFLD.

On the contrary, results of the study by Mazo et al. [15] did not show a statistically significant association between hypothyroidism, simple steatosis, and NASH. Furthermore, two other recently published studies of healthy participants also confirmed the findings of Mazo and colleagues [6,16].

Eshraghian et al. [6] failed to demonstrate any association between markers of thyroid dysfunction and presence of NAFLD. Despite lower free T3 levels among the NAFLD group, serum TSH, free T4, free T3, and markers of thyroid autoimmunity were not different in the participants with NAFLD and those without NAFLD. However, patients with NAFLD were more likely to have low TSH levels, though hypothyroidism and hyperthyroidism were not associated with NAFLD. The observed changes in TSH and free T3 levels may attribute to alterations in thyroid hormones owing to sick euthyroid syndrome in NAFLD [6].

In our study, TPO-Ab did not show any statistically significant correlations when each group of our study is studied separately, but it was noticed that the only four participants who showed positivity to TPO-Ab were belonging to the NAFLD group and were all IR. On the contrary, regarding the thyroid status of each of them, two of them showed subclinical hypothyroidism, whereas the other two had a normal thyroid status. When the same statistical correlations are applied to the total population of the study, TPO-Abs showed statistically significant correlation with TC levels $(P<0.021)$, TGs levels $(P<0.008)$, LDL levels $(P=0.037)$, fasting blood glucose levels $(P=0.041)$, and fasting insulin levels $(P=0.030)$. The prevalence of IR was higher in participants with positive TPO-Ab (100\%) than in those with negative TPO-Ab (37.2\%). These findings raise the possibility that the previously mentioned statistically nonsignificant correlations that we got when we studied each group separately were falsely nonsignificant owing to small sample size.

Previous studies found a cross-sectional positive association between the presence of TPO-Abs and the levels of TSH within the euthyroid range. Those results were consistent with the presence of TPO-Abs, necessitating a compensatory increase in levels of TSH to maintain the euthyroid state. They also found that $\mathrm{TSH}$ and TPO-Abs can predict the development of hypothyroidism in a general population of participants with all normal levels of TSH at baseline. Incidence of hypothyroidism during 9 years of follow-up was significantly higher in participants with positive TPO-Abs at baseline compared with TPO-Absnegative participants. Both TPO-Abs and TSH level are independent predictors of hypothyroidism, even in participants with a TSH level within the normal laboratory's reference range [17].

The pathophysiologic process behind the relationship of TPO-Abs prevalence and future thyroid disease is complex and not completely understood. TPO is not only known to be the primary enzyme in the synthesis of thyroid hormone, it also serves as a major autoantigen. Moreover, it has been hypothesized that TPO-Abs should not be viewed as one entity as their pathogenic potential might be influenced depending on which epitope of TPO they are binding to. The presence of TPO-Abs is characteristic for autoimmune thyroid diseases, both in hypothyroidism (Hashimoto's thyroiditis) and hyperthyroidism (Graves' disease) [17].

Our study did not show any significant correlation between IR (HOMA IR) and FT3 or FT4. This was in agreement with other studies which reported normal insulin sensitivity in patients with thyroid dysfunctions [18]. No correlation was found between hypothyroidism and insulin sensitivity in patients with overt hypothyroidism, based on the assessment of the HOMA IR index [18]. However, it should be noted that fasting hyperinsulinemia was reported and was interpreted as an early sign of impairment of glucose metabolism [18]. On the contrary, it was reported that thyroid disorders, including both hypothyroidism and hyperthyroidism, have been associated with IR owing to various mechanisms such as altered insulin secretion and lipid levels [5]. HOMA values were reported to be significantly higher in clinical hyperthyroidism and hypothyroidism groups as compared with the euthyroid group. Insulin levels were also found to be significantly increased in both groups as compared with the euthyroid group [5]. IR or hyperinsulinemia was also reported to be associated with overt or subclinical hypothyroidism and metabolic syndrome [19]. IR was also found to be significantly higher in the patients with subclinical hypothyroid [19]. Tuzcu et al. showed that subclinical hypothyroidism has been associated with fasting hyperinsulinemia; however, no 
difference was found regarding HOMA IR [20].The main limitations of our study were the small sample size and the fact that the study groups were not age matched. Diagnosis of NAFLD was based on ultrasonography only, and no liver biopsy was done. Our study is also a cross-sectional analytical study and lacks the temporal relationship.

In conclusion, our study showed increased thyroid dysfunction and IR in nondiabetic patients with NAFLD. Further studies are needed to explore the role of thyroid autoimmunity in this relationship.

\section{Financial support and sponsorship}

Nil.

\section{Conflicts of interest}

There are no conflicts of interest.

\section{References}

1 Rinella M. Nonalcoholic fatty liver disease: a systematic review. JAMA 2015; 313:2263-2273.

2 Utzschneider K, Kahn S. The role of insulin resistance in nonalcoholic fatty liver disease. J Clin Endocrinol Metab 2006; 91:4753-4761.

3 Hazlehurst J, Tomlinson J. Mechanisms in endocrinology: Non-alcoholic fatty liver disease in common endocrine disorders. Eur J Endocrinol 2013; 169:R27-R37.

4 Chalasani N, Younossi Z, Lavine J, Diehl A. The diagnosis and management of non-alcoholic fatty liver disease: practice guideline by the American Gastroenterological Association, American Association for the Study of Liver Diseases, and American College of Gastroenterology. Hepatology 2012; 142:1592-1609.

5 Kapadia K, Bhatt P, Shah J. Association between altered thyroid state and insulin resistance. J Pharmacol Pharmacother 2012; 3:156-160.

6 Eshraghian A, Dabbaghmanesh M, Eshraghian H, Fattahi M, Omrani G. Nonalcoholic fatty liver disease in a cluster of Iranian population: thyroid status and metabolic risk factors. Arch Iran Med 2013; 16:584-589.
7 Ahuja V, Kadowaki T, Evans RW, Kadota A, Okamura T, El Khoudary SR, et al. Comparison of HOMA-IR, HOMA- $\beta \%$ and disposition index between US white men and Japanese men in Japan. Diabetologia 2015; 58:265-271.

8 Pagano G, Pacini G, Musso G, Gambino R, Mecca F, Depetris N, et al. Nonalcoholic steatohepatitis, insulin resistance, and metabolic syndrome: further evidence for an etiologic association. Hepatology 2002; 35:367-372.

9 Patrik L. Nonalcoholic fatty liver disease: relationship to insulin sensitivity and oxidative stress. Treatment approaches using vitamin E, magnesium, and betaine. Altern Med Rev 2002; 7:276-292.

10 Bugianesi E. Review article: steatosis, the metabolic syndrome and cancer. Aliment Pharmacol Ther 2005; 22 (Suppl 2):40-43.

11 Bugianesi E, Gastaldelli A, Vanni E. Insulin resistance in non-diabetic patients with non-alcoholic fatty liver disease: sites and mechanisms. Diabetologia 2005; 48:634-642.

12 Ludwig U, Holzner D, Denzer C, Greinert A, Haenle M, Oeztuerk S, et al. Subclinical and clinical hypothyroidism and non-alcoholic fatty liver disease: a cross sectional study of a random population sample aged 18 to 65 years. BMC Endocr Disord 2015; 15:41.

13 Pagadala M, McCullough A. Editorial: non-alcoholic fatty liver disease and obesity: not all about body mass index. Am J Gastroenterol 2012; 107:1859-1861.

14 Pagadala M, Zein C, Dasarathy S, Yerian L, Lopez R, McCullough A. Prevalence of hypothyroidism in nonalcoholic fatty liver disease. Dig Dis Sci 2012; 57:528-534.

15 Mazo D, Lima V, Stefano J, Rabelo F, Faintuch J, Oliveira C. Gluco-lipidic indices in treated hypothyroidism associated with nonalcoholic fatty liver disease. Arq Gastroenterol 2011; 48:186-189.

16 Ittermann T, Haring R, Wallaschofski H, Baumeister S, Nauck M, Dörr M, Völzke $H$. Inverse association between serum free thyroxine levels and hepatic steatosis: results from the Study of Health in Pomerania. Thyroid 2012; 22:568-574.

17 Roos A, Links T, de Jong-van den Berg LT, Gans LT, Wolffenbuttel BH, Bakker SJ. Thyroid peroxidase antibodies, levels of thyroid stimulating hormone and development of hypothyroidism in euthyroid subjects. Eur $\mathrm{J}$ Intern Med 2010; 21:555-559.

18 Gierach M, Gierach J, Junik R. Insulin resistance and thyroid disorders. Endokrynol Pol 2014; 65:70-76.

19 Cheserek M, Wu G, Shen L, Shi Y, Le G. Evaluation of the relationship between subclinical hypothyroidism and metabolic syndrome components among workers. Int J Occup Med Environ Health 2014; 27:175-187.

20 Tuzcu A, Bahceci M, Gokalp D, Tuzun Y, Gunes K. Subclinical hypothyroidism may be associated with elevated high-sensitive Creactive protein (low grade inflammation) and fasting hyperinsulinemia. Endocr J 2005; 52:89-94 